\title{
Dental Age Estimation: A Review
}

\author{
Donni S ${ }^{1,2}$, Haslinda R ${ }^{3}$, Phrabhakaran N ${ }^{4}$, Aspalilah $\mathrm{A}^{1 *}$ \\ ${ }^{1}$ Department of Basic Sciences and Oral Biology, Faculty of Dentistry, Universiti Sains Islam Malaysia. \\ ${ }^{2}$ Department of Primary Care, Faculty of Dentistry, SEGI University. \\ ${ }^{3}$ Department of Periodontology and Community Health, Faculty of Dentistry, Universiti Sains Islam Malaysia. \\ ${ }^{4}$ Department of Oral Biology, Faculty of Dentistry, MAHSA University.
}

${ }^{*}$ Correspondence author: Dr. Aspalilah Alias, Senior Lecturer, Department of Basic Sciences and Oral Biology Faculty of Dentistry, Universiti Sains Islam Malaysia, Block B Level 15, Persiaran MPAJ, Jalan Pandan Utama, 55100 Kuala Lumpur; Tel: +60342891154; Email: draspa76@usim.edu.my

Received: October 07, 2018; Accepted: October 12, 2018; Published: October 12, 2018;

\begin{abstract}
Age is one of the essential factors in establishing the identity of the person. It plays an important role in forensic medicine, clinical dentistry and archaeology. Age estimation is crucial in medico legal cases and importance in forensic medicine, not only for identifying deceased victims but also in connection with crimes and accidents. Age estimation of unknown human bodies is very important in the setting of a crime investigation as well as mass disaster. The age for the individual can be assessed as skeletal, morphological, secondary sex character and dental age.This paper reviews various dental age estimation methods that have been used by the scientist in order to help the identification of remains.
\end{abstract}

Keywords: Age Estimation, Identification, Forensic Dentistry

\section{Introduction}

Age is one of the important biological profiles in the individuals. Thus far many anthropologists have studied the age systems, where age is often a major organizing principle. Age systems include formal age classes of individuals of similar numerical age, age grades or developmental stages based on social and biological development, and relative ages of individuals [1].

In many cases, chronological age and biological age may not be the same, due to the developmental variations. Hence, different parameters such as dental age, bone age, mental age, and other factors such as menarche, voice change, height, and weight are considered as the proxy indicator for biological age and body development [2]. Dental development is more reliable as an indicator of biological maturity in children. Dental maturity is more relevant as it is less affected by nutritional and endocrine status [2].

Over the past 100 years, forensic dentistry has become a part of forensic sciences that utilizes dental or orofacial findings in the legalistic system. The fundamental role of forensic dentistry in identification of human remains has been proved by many researchers in their studies [3]. There has been strong relationship between the growth rate of bone and teeth, which can be utilized for age identification of an individual. Tooth formation is used often to assess maturity and predict age. Within clinical dentistry, this information aids in diagnosis and treatment planning [4].

Various factors are considered for determination of age, out of which teeth are the most durable structures in the human body which are better preserved even in the acidic soil. Human teeth are the hardest substances in the human body and depending upon ambient conditions, characteristics associated with the teeth may provide an important and effective method to identify a person. Dental tissues are among the most durable tissues of the human body resistant to different external influence, as well as to mechanical, thermal and chemical irritations [5]. Thus, this reviewed paper was performed to determine various dental age estimation methods that have been used by the scientist in order to help the identification of individuals.

\section{Methods of Age Determination}

Many literatures describe various methods or techniques that address dental age estimation in human. The methods can be classified as follows:

\section{Based on Tooth Development}

Age estimation using tooth can be classified into two periods of time in human life. The first period is when the teeth are developing in the jaws up to 20 years of age. The second period when all teeth are fully formed [6].

\subsection{Age estimation of individuals below 20 years of age}

Most scientific studies have been developed developmental tables for the different stages of tooth formation [6]. These methods based upon developmental stages, are more accurate than age estimation based on the stages of eruption of teeth [6]. In these age periods, in many cases the statistical scientific tables have been used due to its stronger evidence and more accurate technique than visual age assessment [6].

However, the dental development may be decelerated by severe and long lasting diseases such as congenital syndromes and also 
nutritional deficiencies. On the other hand, only rare hormonal hyper secretion may accelerate the development [6]. Severe dental diseases and tooth extraction may also influence the dental development [6]. For a most accurate assessment of the age it is necessary to assess these factors and take them into consideration.

For an example, study had done by Solheim and Vonen, they used at least two different age estimation techniques in cases of age estimation in individual below 20 years of age. Generally they use tables from Finland of Haavikko [7] and from Canada by Anderson et al [8].

\subsection{Age estimation of individuals above 20 years of age}

For this group, age estimation relies upon regressive age changes such as attrition, loss of periodontal attachment and secondary dentin formation. In this case the visual assessment may be almost as accurate as the calculated age according to a specific technique. A visual assessment may thus be an important supplement to scientific methods [6].

The pioneer scientist who had been created the technique for age estimation was Gustafson [9]. In this method, longitudinal sections of teeth cut through the central area was examined. The age-related biological changes such as attrition, periodontal ligament retraction, cementum apposition, secondary dentin formation, root resorption and transparency of the root were scored from 0 to 3 . The scores were added and a regression analysiswas performed. The formulae based upon regression analysis e.g multiple regressions with age as dependent variable and the different age related changes as independent variables [9]. However, this method was modified due to ethics involvement extract teeth in living person. Thus other techniques such as Radiographic methods is one can be used. It is based on the size of the pulp in the relation to the whole tooth and gives a measure of the secondary dentin formation [10].

The other morphologic technique in living person is methods by Solheim. This technique is based only upon attrition, colour and recession of the periodontal attachment. All these variables can be assessed in a living person [11].

\section{Based on Dentition}

\subsection{Pre natal, neonatal and post natal}

Age estimation in this group of individuals can be very accurate. Histological methods are used to assess the stage of tooth development during the pre-mineralization period. Mineralization of deciduous dentition commences from two or four months in-utero [12]. Some of the histological methods can detect early mineralization 12 weeks before being detectable in the radiographs. Before the mineralization of tooth germs starts, the tooth germs may be visible as radiolucent areas on the radiograph; the subsequent radiographs of the mandible will depict the deciduous teeth in various stages of mineralization as per the pre-natal age of the fetus [12].

\subsection{Children and adolescents}

In this stage, the age estimation is based on the time of emergence of the tooth in the oral cavity and the tooth calcification. The radiographic analysis of developing dentition, especially when there is no clinical evidence available (2.5-6 years) as well as the clinical tooth emergence in various phases will help in age determination. Schour and Massler's chart was the first attempt to study dental age estimation [12]. This chart permits direct comparisons with radiographs. Demirjian et al developed an age estimation method that made use of a scoring system. In this method, seven mandibular teeth on the left side were divided into 8 stages and maturity score was evaluated [13]. Age estimation also can be measured using mandibular third molars in which formed part of root were digitized but the precision of the age estimation was slightly inferior compared with the standard method [14].

\subsection{Adults}

Most of the methods used in adults use various regressive changes of hard and soft tissues of the teeth [3,5,9-11]. Gustafson (1950) studied the changes occurring in individual teeth and succeeded in estimating the age with some accuracy. He used 6 dental changes connected with aging namely, attrition, apical migration of periodontal ligament, deposition of secondary dentin, cemental opposition, root resorption and transparency of the root dentin. Age was estimated using the formula. It was found that an increase in the total score corresponds to an increase in age [9].

\section{Based on The Methods}

\subsection{Morphological methods}

Morphological methods are based on assessment of teeth (exvivo). Hence, these methods require extracted teeth for microscopic preparation [9]. However, these methods may not be acceptable due to ethical, religious, cultural, or scientific reasons. Gustafson (1950), Dalitz (1962), Bang and Ramm (1970), Johanson (1971), Maples (1978), Solheim (1993) are few scientist that use morphological methods [15].

\subsection{Biochemical}

The biochemical methods are based on the racemization of amino acids. The racemization of amino acids is a reversible first-order reaction and is relatively rapid in living tissues in which metabolism are slow. Aspartic acid has been reported to have the highest racemization rate of all amino acids and to be stored during aging. In particular, L-aspartic acids are converted to D-aspartic acids and thus the levels of $\mathrm{D}$-aspartic acid in human enamel, dentine, and cementum increase with age. Assessment of age using this method was first described by Helfman and Bada in 1975 [15].

\subsection{Radiographic}

Radiographic plays an important role in the human age determination. Radiographic images are utilized in the process of age estimation, which is one of the essential tools in identification in forensic science $[3,10-12,16]$. The assessment of age with this method is simple, non-invasive procedure and reproducible that can be employed both, on living and unknown dead. $[10,12,16]$.

Various radiographic techniques that can be used in age identification are intraoral periapical radiographs, lateral oblique 
radiographs, cephalometric radiographs, panoramic radiographs, digital imaging and advanced imaging technologies $[10,12,16]$.

\section{Conclusion}

Age estimation in human provides a comprehensive issue. Consequently, needs considerable experience in recognizing significant changes and allowing for their variability within any particular population. Human teeth are particularly helpful in age estimation because can display a number of observable ages related variables. Teeth also tend to remain intact under circumstances compare other parts in the human body which might alter or obliterate the rest of the skeleton.

\section{References}

1. Smith T, Brownless L (2011) Age assessment Practices: A Literature Review \& Annotated Bibiliography. New York: United Nations Children's Fund (UNICEF).

2. McKenna CJ, James H, Taylor JA, Townsend GC (2002) Tooth development standards for South Australia. Aust Dent J 47: 223-227. [crossref]

3. Limdiwala PG, Shah JS (2013) Age estimation by using dental radiographs. $J$ Forensic Dent Sci 5: 118-122. [crossref]

4. Briffa K, Dougall NB, Galea J, Mifsud D, Camilleri S (2005) Chronologic and Dental Ages of Maltese Schoolchildren - A pilot Study. Malta Medical Journal 17: 31-35.

5. Gupta P, Kaur H, Shankari G S M, Jawanda MK, Sahi N (2014) Human age estimation from tooth cementum and dentin. J Clin Diagn Res 8: ZC07-10. [crossref]

6. Solheim T, Vonen A (2006) Dental Age Estimation - Quality Assurance and Age Estimation of Asylum Seekers in Norway. Forensic Science International 4747: $56-60$.

7. Haavikko K (1970) The formation and the alveolar and clinical eruption of the permanent teeth. An orthopantomographic study. Suom Hammaslaak Toim 66: 103-170. [crossref]

8. Anderson DL, Thompson GW, Popovich F (1976) Age of attainment of mineralization stages of the permanent dentition. J Forensic Sci 21: 191-200. [crossref]

9. GUSTAFSON G (1950) Age determination on teeth. $J$ Am Dent Assoc 41: 45-54. [crossref]

10. Kvaal SI, Kolltveit KM, Thomsen IO, Solheim T (1995) Age estimation of adults from dental radiographs. Forensic Sci Int 74: 175-185. [crossref]

11. Solheim T (1993) A new method for dental age estimation in adults. Forensic Sci Int 59: 137-147. [crossref]

12. Panchbhai AS (2011) Dental Radiographic Indicators, A Key to Age Estimation. DentoMaxillofacial Radiology 40: 199-212.

13. Willems G, Van Olmen A, Spiessens B, Carels C (2001) Dental age estimation in Belgian children: Demirjian's technique revisited. J Forensic Sci 46: 893-895. [crossref]

14. Wedl JS, Friedrich RE (2005) [Measuring the distance of the wisdom teeth from the occlusal plane as forensic-odontological method for chronological age determination]. Arch Kriminol 215: 77-84. [crossref]

15. Priyadarshini C, Puranik MP, Uma SR (2015) Dental age Estimation Methods: A Review International Journal of Advanced Health Sciences 1(12).

16. Karaarslan B, Karaarslan ES, Ozsevik AO, Ertas E (2010) Age estimation for Dental Patients Using Orthopantomographs. European Journal of Dentistry 4: 389-394.

Citation:

Aspalilah Alias (2018) Dental Age Estimation: A Review. J Dent Maxillofacial Res Volume 1(1): 1-3. 